\title{
A Comparative Study of Deep Learning Models With Handcraft Features and Non-Handcraft Features for Automatic Plant Species Identification
}

\author{
Shamik Tiwari, UPES University, India
}

\begin{abstract}
The classification of plants is one of the most important aims for botanists since plants have a significant part in the natural life cycle. In this work, a leaf-based automatic plant classification framework is investigated. The aim is to compare two different deep learning approaches named Deep Neural Network (DNN) and deep Convolutional Neural Network (CNN). In the case of deep neural network, hybrid shapes and texture features are utilized as hand-crafted features while in the case of the convolution non-handcraft, features are applied for classification. The offered frameworks are evaluated with a public leaf database. From the simulation results, it is confirmed that the deep CNN-based deep learning framework demonstrates superior classification performance than the handcraft feature based approach.
\end{abstract}

\section{KEYWORDS}

CNN, Deep Learning, Feature Extraction, Plant Classification

\section{INTRODUCTION}

Plants are an essential part of environment and important for human being. Plant species are valuable as medicine, as foodstuff and also for industrial applications. Identifying plant species helps to ensure the survival and protection of all natural life (Ehsanirad and Kumar, 2010). Classification is very important when it comes to taxonomy. As a result, such categories of species can be grouped or clustered to a specific location. Recognition of plant classes has many benefits comprising identification of medicinal plants used in ayurvedic medicines as well as other uses (Raal and Sõukand, 2005).

Leaves are suitable part of plants to categorize plant species since they are more readily accessible than the other parts of plant like flowers that are obtainable for a small period. Leaf recognition is based on different characters presented on the leaf surface. These characters will determine the class to which an individual plant species belong. Botanists to categorize particular plant species have followed the leaf recognition technique. Usually, plants have distinctive leaf properties that make them dissimilar from each other in a large number of appearances like texture, shape, colour, and the size (Priya et al., 2012). 
Leaf recognition is limited to factors like climate and plant health because the external surface of the leaf can have change in colour due to diseases or climate changes. This makes it difficult the job of identification. This process is also tedious since human intelligences for plant identification are inadequate for large number of species. Over last decades, various Computer-Aided Detection (CAD) methods are suggested to increase leaf based plat recognition rate (Du et al., 2013). Primarily, handcraft features based recognition approaches are offered in last decades. This work presents the use of handcraft features for plant species detection.

These days, deep learning frameworks based on convolutional neural network have used successfully in many object identification uses. CNN classification model does not require handcraft features for object recognition. Here, convolution layer achieves the job of feature identification (Shin et al., 2016). CNN based classification model is also proposed for plant identification in this work. Finally, a comparison of handcraft features and automatic feature extraction using two different deep learning models is discussed. Rest of the paper is distributed into nine sections. Section 2 discusses prominent works in the area as literature review. Deep learning models are discussed in Section 3. Proposed methodology is discussed in Section 4. Handcraft features and leaf database is discussed in sections 5 and 6 respectively. The details of experiments and results is given in Section 7. As a last section, conclusion is discussed in Section 8.

\section{LITERATURE REVIEW}

A considerable work is proposed in the area of leaf-based plant recognition during the last decade using image processing and computer vision methods. Kadir et al. (2011) have presented a probabilistic neural network-based classification model for 60 types of foliage plants. This work has used mean, standard deviation, skewness as colour moments, shape features, grey level co-occurrence matrixbased texture features and Polar Fourier Transform. Vein features also added to increase the accuracy of the system. The result displays that the system provides mean accuracy of $93.0833 \%$. In a similar type of another work, Kadir et al. (211) have utilized shape, texture, vein and colour features for plant classification Flavia dataset. This dataset consists of 32 types of leaves. A neural network model is designed with these features. The average accuracy of $93.75 \%$ is attained in this work.

A plant classification method established on Random Forest Regression (RFR) and Linear Discriminant Analysis (LDA) is proposed by Elhariri et al. (2014). A combination of features namely HSV colour moments, shape, grey level co-occurrence matrix, vein features and first order texture, are used for both the classifiers. Experimental results have demonstrated that LDA reached better categorization accuracy of $92.65 \%$ than the random forest method that attained classification accuracy of $88.82 \%$. Haque et al. (2018) have presented a work for plant classification that uses seven leaf features extracted from geometric features of leaf shape after pre-processing of leaf images. A straightforward concept of minimum Euclidean distance is utilized for achieving the recognition. This scheme have used 10 species of leaves from the Flavia image dataset and gained classification accuracy around 90\%. Riaz et al. (2018) have designed an ensemble classifier for leaf identification for plant species categorization. This ensemble classifier utilizes morphological, Zernike moments and shape features. In this work, maximum $87 \%$ accuracy is achieved by a combination of morphological features and textural.

A deep learning-based model is most popular these days, particularly for image classification. GoogLeNet as a deep learning model is used to classify 50 types of Chinese herbal plants under usual circumstances with complex backgrounds is presented by Liu et al. (2018). An average accuracy up to $89.4 \%$ is attained in this work. Lee et al. (2017) have designed CNN model to identify leaf features straight from the raw leaf images, and gain insight of the selected features relies on a De-convolutional Network (DN) method. Convolutional neural network (sometimes referred as ConvNet) models are also designed to achieve plant disease recognition and diagnosis with the leaves of fit and unhealthy plants 
by Ferentinos (2018). Total 58 dissimilar classes of plant and disease combinations are considered for 25 plant species. The designed model has attained $99.53 \%$ accuracy.

A CNN-based D-Leaf model projected by Tan et al. (2018). Initially, features are extracted by three separate convolution networks i.e. AlexNet, tuned AlexNet and D-Leaf from leaf images. Later, these features are classified by utilizing multiple classification models, namely, Support Vector Machine (SVM), Artificial Neural Network (ANN), Naïve-Bayes (NB), k-Nearest-Neighbour (K-NN) and CNN. The D-Leaf model achieved $94.88 \%$ accuracy, which is highest among all the models.

\section{DEEP LEARNING}

Deep Learning is a part of machine learning procedures motivated by the configuration and function of Artificial Neural Networks (ANN). Deep learning approaches target at learning feature hierarchies with higher-level features formed by the alignment of low-level features. The learning comprises two main phases (Li et al., 2015):

- The first step applies a nonlinear transformation of the input and build a statistical model as output;

- The second step targets at refining the model with a derivative approach.

The network iterates these two steps multiple time until it has touched an acceptable level of accuracy. There are multiple deep learning models are available depending on applications. Two most commonly deep learning architectures are used in this work. Details of these models are given following subsections.

\subsection{Deep Neural Network (DNN)}

Deep learning falls in the domain of artificial neural networks that has the neural networks with more than three layers, i.e., one input layer, one output layer and more than one hidden layer. The term DNN is refer to the neural networks designed with deep architecture. DNN is proficient of learning complex features with high density and abstraction than lighter neural networks. DNNs arise in a large range of shapes and sizes subjective to the use. The common sizes and shapes are also growing speedily to increase precision and efficiency. The input to these networks is a set of values characterizing the facts to be analysed in all cases.

\subsection{Convolution Neural Network (CNN)}

$\mathrm{CNN}$ is specialized type of neural network mostly deals with images. It is the most efficacious model in artificial intelligence. The organization of CNN usually consists of four different sorts of layers. A CNN comprises convolutional layer, activation layer, pooling layer and a fully connected layer (Tiwari, 2016).

The major aim of convolution layer is feature extraction from the input image. The convolution mathematical operation is performed in this layer. In continuous case convolution of two functions $f$ and $g$ is given by:

$$
\left(f^{*} g\right)(t)=\int_{-\infty}^{\infty} f(\tau) g(t-\tau) d \tau=\int_{-\infty}^{\infty} f(t-\tau) g(\tau) d \tau
$$

In discrete case, the equivalent convolution operation is given by:

$$
\left(f^{*} g\right)(n)=\sum_{m=-\infty}^{\infty} f(m) g(n-m)=\sum_{m=-\infty}^{\infty} f(n-m) g(m)
$$


This 1-D convolution can be extended to 2-D convolution for digital image as:

$$
\left(f^{*} g\right)(x, y)=\sum_{m=-M}^{M} \sum_{n=-N}^{N} f(x-n, y-m) g(n, m)
$$

In this case the function $g$ is referred as filter or kernel applied to input image $f$.

The principle of $2 \mathrm{D}$ convolution is to move a convolution filter on the input image. The filter (kernel) moves by a number of pixels that is termed as stride. At each position, the convolution between the filter and the part of the image is achieved. The result is a two-dimensional array referred as feature map. After getting the feature map, it is passed through a nonlinear activation layer, such as softmax, Rectified Linear Unit (ReLU), Randomized Leaky ReLU, Leaky ReLU, Parameterized ReLU, and Exponential Linear Units (ELU) etc. Activation layer in the form of activation function determines whether a neuron should be triggered or not by computing weighted sum and supplementary adding bias with it. The determination of the activation function is to present non-linearity to the neuron's output. Spatial Pooling also known as subsampling or down sampling decreases the dimensionality of each feature map however preserves the most significant information. It also controls overfitting by reducing the number of parameters and computations. Pooling can be of different types like max, average and sum etc. the last layer is fully connected layer or a dense layer. It is referred as dense layer since each neuron obtains input from all the neurons in the earlier layer, thus fully connected. This layer gives the final output of the model (Shin et al., 2016).

\section{PROPOSED METHODOLOGY}

Two different methodologies are utilized for leaf-based plant classification. First methodology utilises a traditional model of classification as shown in Figure 1. In this traditional model, handcraft features are calculated from pre-processed leaf images as discussed in Section 5. These features are then feed to deep neural network for classification. The second methodology for leaf classification does not utilise these handcrafted features since this is based on CNN model as shown in Figure 2. In case

Figure 1. The DNN model of classification

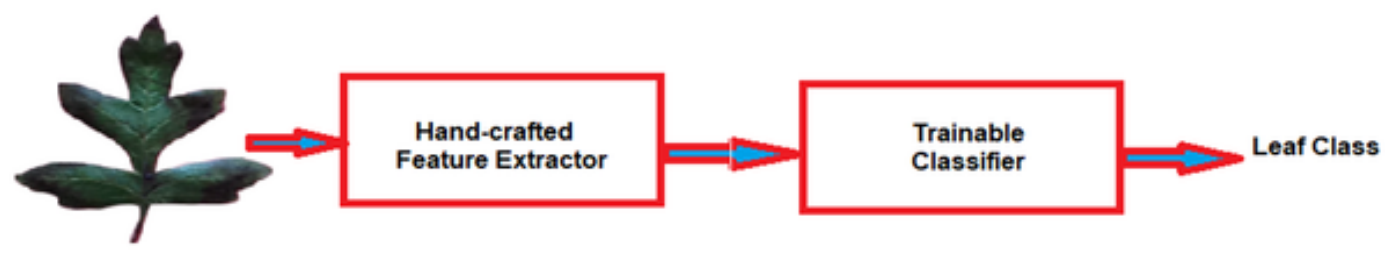

Figure 2. The CNN model of classification

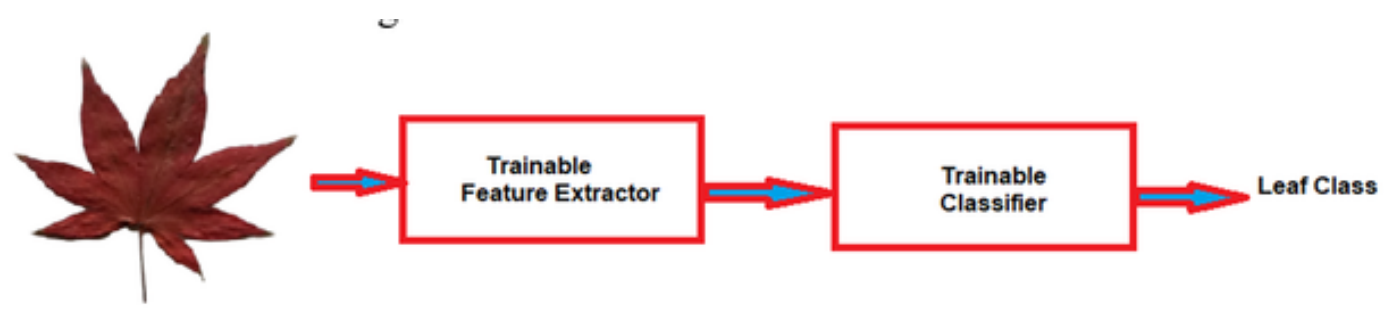


of CNN model, it is the job of convolution layer to identify the leaf features. The segmented RGB images are passed to CNN model for classification. Finally, the performance of the both approached is compared using performance metrics.

\section{HANDCRAFT FEATURE SET}

Shape based and textures features are considered as the hybrid feature set for classification using deep neural network. The eccentricity $\left(E_{c}\right)$, aspect ratio $\left(A_{R}\right)$, elongation $\left(E_{G}\right)$, solidity $(S)$, stochastic convexity $\left(S_{C}\right)$, isoperimetric factor $\left(I_{F}\right)$, maximal indentation depth $\left(M_{I D}\right)$ and lobedness $(L)$ are calculated as shape based features while mean intensity $(m)$, mean contrast $(\sigma)$, smoothness $\left(S_{m}\right)$, third moment $\left(\mu_{3}\right)$, uniformity $(U)$ and entropy $(E)$ are calculated as texture features. Therefore, hybrid feature set consists of total 14 features. The description of each feature is given below.

\subsection{Shape Based Features}

Shape is a significant visual feature and widely acceptable basic feature for object description. Following eight shape-based features are calculated from segmented leaf images (Chaki and Parekh, 2011):

1. Eccentricity $\left(\boldsymbol{E}_{c}\right)$ : The proportion of the major axis length and distance between the foci of the ellipse. Eccentricity is able to distinguish long and rounded leafs;

2. Aspect Ratio $\left(\boldsymbol{A}_{\boldsymbol{R}}\right)$ : The proportion between the extreme length $\operatorname{Lmax}$ and the least length Lmin of the least bounding rectangle:

$$
A_{R}=\frac{\operatorname{Lmax}}{\operatorname{Lmin}}
$$

3. Elongation $\left(\boldsymbol{E}_{G}\right)$ : Elongation is further notion founded on eccentricity. Elongation is a feature that receives values in the range $[0,1]$. A regular contour in all axes such as a square or circle has an elongation value of 0 while shapes with high aspect ratios has an elongation factor nearer to 1 . It is defined as follows:

$$
E_{G}=1-\frac{2 D \max (O)}{D \min (O)}
$$

where $\operatorname{Dmax}(O)$ is the diameter of the prime inscribed circle and $\operatorname{Dmin}(O)$ is the diameter of the minimum circumscribed circle for the object $O$.

4. Solidity ( $\boldsymbol{S})$ : The proportion between object's region and region of the object's convex hull, where region is measured in terms of area. It can also be considered as a parameter for convexity of object:

$$
S=\frac{\operatorname{Area}(O)}{\operatorname{Area}(C(O))}
$$


where Area $(O)$ is the area of object and Area $(C(O))$ is the area of convex hull for the object.

5. Stochastic Convexity $\left(S_{C}\right)$ : This feature lengthens the standard convexity in topological sense, by using performing the sampling in object area. It measures the probability of a randomly selected region $S(x, y), x, y \in O$ to be the part of object $O$;

6. Isoperimetric Factor ( $\boldsymbol{I}_{F}$ ): Isoperimetric factor show a high-level correlation with the solidity, elongation and maximal indentation depth. It is an eminent shape characteristic, described by:

$$
I_{F}=\frac{4 \pi \cdot \operatorname{Area}(O)}{L(\partial(O))^{2}}
$$

where $\operatorname{Area}(O)$ is the area of the object and $L(\partial(O))$ is the length of contour for object $O$.

7. Maximal Indentation Depth $\left(\boldsymbol{M}_{I D}\right)$ : The depth from the surface of the object to the indentation depth of the indenter or indenter slope is referred as indent depth. The maximum depth is the overall shift of the object and the indenter at maximum weight that comprises the elastic deformation. Let $L(C(O)), X_{C(O)}$ and $\delta I$ represents the arc length, centroid of the convex hull and boundary of the object $O$ in that order. The distances $d\left(x, X_{C(O)}\right)$ and $d\left(y, X_{C(O)}\right)$ are calculated for $\forall x \in C(O)$ and $\forall y \in \delta I$. The indentation depth can be defined as:

$$
M_{I D}=\frac{\left[d\left(x, X_{C(O)}\right)-d\left(y, X_{C(O)}\right)\right]}{L(C(O))}
$$

The indentation depth is sampled with interval of one degree and peak value of this function is referred as maximal indentation depth.

8. Lobedness $(\boldsymbol{L})$ : By differentiating between lobed and unlobed leaves, shape-based leaf recognition methods can be enriched. It is achieved by computing the number of inflection points in the contour distance signature. The mean is subtracted before the Fourier Transform of the indentation function is computed. The received spectrum is standardized by the total energy. It is calculated as:

$$
L=F_{m i n}(u, v) X D^{2}
$$

where $F_{\min }(u, v)$ represents the least frequency at which the cumulated energy surpasses $80 \%$.

\subsection{Texture Features}

Texture is an iterating pattern of local variations in gray levels. It is an important clue for the analysis of many images. It is generally applied to identify intrinsic characteristics of surfaces particularly those that do not have a smoothly varying intensity. Following six texture features are used for leaf 
images. Let $z$ be a random variable representing intensity levels and let $p\left(z_{i}\right), i=0,1,2,3, \ldots, L-1$, be the corresponding histogram, where $L$ is the count of distinct grey levels (Gaur et al., 2014):

1. Mean Intensity ( $\boldsymbol{m}$ ): It represents the average intensity of the leaf image:

$$
m=\sum_{i=0}^{L-1} z_{i} \cdot p\left(z_{i}\right)
$$

2. Mean Contrast $(\sigma)$ : Contrast is an extent of the local variations available in leaf image. It is the standard deviation of the grey image:

$$
\sigma=\sqrt{\sum_{i=0}^{L-1}\left(z_{i}-m\right)^{2} p\left(z_{i}\right)}
$$

3. Smoothness $\left(S_{m}\right)$ : It represents the relative smoothness of the grey levels in the object's region. It is calculated as:

$$
S_{m}=\frac{1}{1+\sigma^{2}}
$$

where $\sigma^{2}$ is the variance or second moment of the gy level image.

4. Third moment $\left(\mu_{3}\right)$ : The $3^{r d}$ moment of $z$ about the mean $(m)$ is defined as:

$$
\mu_{3}(z)=\sum_{i=0}^{L-1}\left(z_{i}-m\right)^{3} p\left(z_{i}\right)
$$

It measures skewness of the histogram.

5. Uniformity ( $\boldsymbol{U})$ : One more valuable texture feature based on histogram is the uniformity that is defined as:

$$
U=\sum_{i=0}^{L-1} p^{2}\left(z_{i}\right)
$$

Uniformity has the maximum value for an image with same grey levels for all pixels i.e. maximally uniform and reduces from there.

6. Entropy ( $\boldsymbol{E})$ : It is an additional significant measure of variability that is educed from basic information theory. It calculates the average randomness of grey levels in image. The value of entropy is zero for an image with constant grey levels. Entropy is defined as: 


$$
E=-\sum_{i=0}^{L-1} p\left(z_{i}\right) \log _{2} p\left(z_{i}\right)
$$

Finally, the handcraft feature vector $F$ consists of these 14 features, which are used for leaf classification using DNN model. Therefore:

$$
F=\left\{E_{c}, A_{R}, E_{G}, E, S, I_{F}, M_{I D}, L, m, \sigma, S_{m}, \mu_{3}, U, E\right\}
$$

\section{LEAF IMAGE DATASET}

The considered leaf image and feature dataset is proposed by Silva et al. (2014). This image dataset has 340 leaf images of 40 different plants. The images have the size of $720 \times 960$ pixels. All the images are 24 bit RGB, and captured with a different background. Leaf classes from 1 to 15 and from 22 to 36 exhibit straight leaf structure and classes from 16 to 21 and from 37 to 40consist of complex leaf structures. Therefore, only 30 dissimilar leaf classes are considered in this work. A sample leaf image for each class of considered plant species is given in Figure 3. The number of images in these leaf classes are $12,10,10,8,12,8,10,11,13,14,16,12,13,12,10,10,12,11,13,9,12,11,12,12,12$, $11,11,11,11,11$, and 10 in the same order as they appear in Figure 3 . This dataset also provides the shape and texture feature separately for each class of image as discussed in Section 5. These features are also used in this work for the same 30 classes as discussed above.

\section{EXPERIMENT AND RESULTS}

To separate experiment are carried out for plant species classification. In the first experiment the shape and texture features and DNN are used as discussed in subsection 7.1. The second experiment uses segmented leaf images to train and test CNN model as discussed in subsection 7.2. To evaluate the performance of classification models, some widely acceptable statistical metrics are used. The description of each metric is as follows (Tiwari, 2017):

- Precision: Precision specifies that what percentage of positive classification are true:

$$
\text { Precision }=\frac{\text { True Positive }}{[\text { True Positive }+ \text { False Positive }]=\text { Total Predicted Positive }}
$$

- Recall: Recall specifies that among the total prediction what percentage of positive prediction are true. It is also termed as sensitivity:

$$
\text { Recall }=\frac{\text { True Positive }}{[\text { True Positive }+ \text { False Negative }]=\text { Total Actual Positive }}
$$

- F-score: F-score is the weighted mean of recall and precision to recall and precision:

$$
F-\text { Score }=2 * \frac{\text { Recall } * \text { Precision }}{\text { Recall }+ \text { Precision }}
$$


Figure 3. Sample leaf images from the database
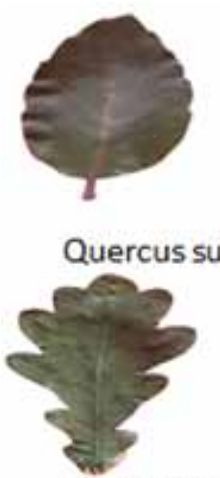

Quercus robur

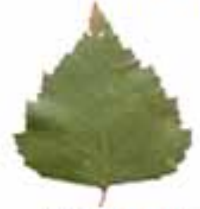

Betula pubescens

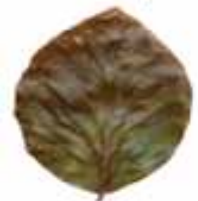

Corylus avellana

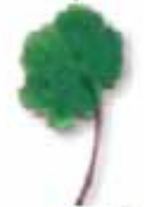

Erodium sp.

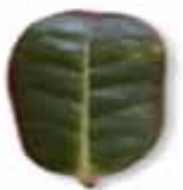

llex perado ssp. azorica

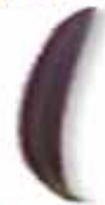

Podocarpus sp.
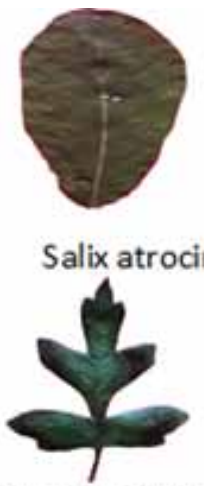

Crataegus monogyna

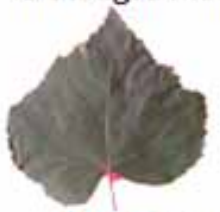

Tilia tomentosa

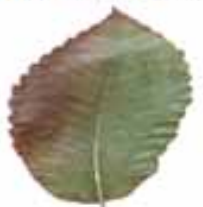

Castanea sativa

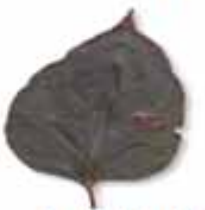

Bougainvillea sp.

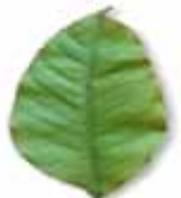

Magnolia soulangeana

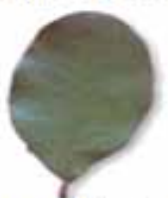

Acca sellowiana

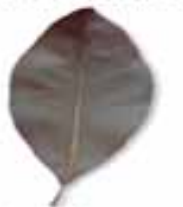

Magnolia grandiora
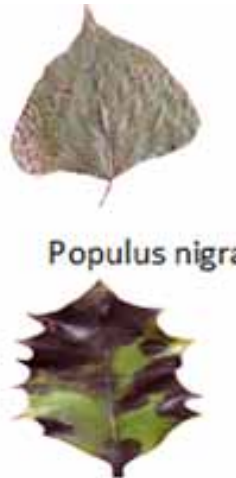

Ilex aquifolium

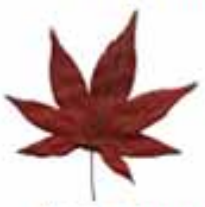

Acer palmatum

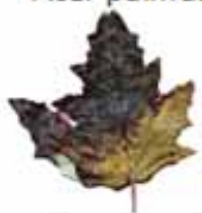

Populus alba

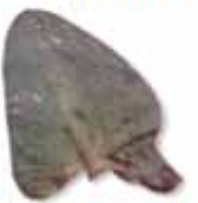

Arisarum vulgare

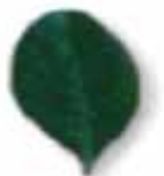

Buxus sempervirens

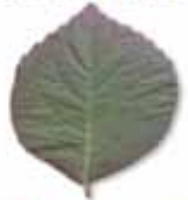

Hydrangea sp.

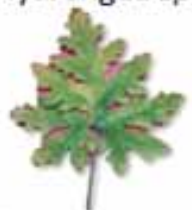

Geranium sp.

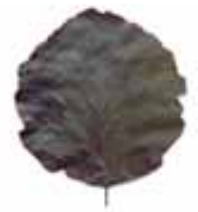

Alnus sp.

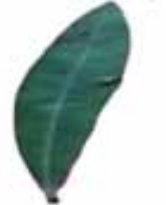

Nerium oleander

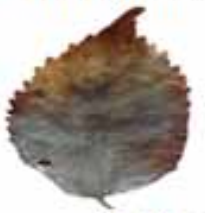

Celtis sp.

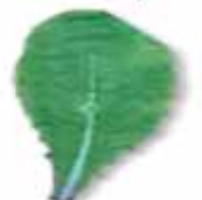

Primula vulgaris

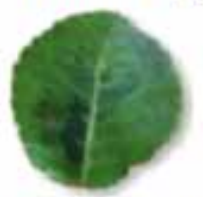

Euonymus japonicus

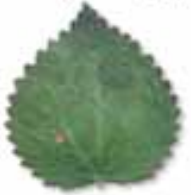

Urtica dioica

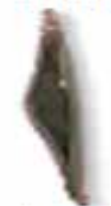

Pseudosasa japonica 


\subsection{Experiment 1 - Handcraft Features Based on Deep Neural Network Leaf Classification Model}

In the first handcraft features based experiment, the standard dense layers are considered. Four dense layers are used in DNN architecture. All four layers are the dense layers with 50, 70, 80 and 6 neurons correspondingly. The first dense layer works as input layer, the second and third dense layers act as hidden layers and the final layer yields the outputs. ReLU is applied as the activation function that has the advantage to overcome from vanishing gradient problem. First three layers are followed by dropout layers to avoid overfitting. The dropout rate of 0.2 is considered in dropout layers. The stochastic gradient descent (sgd) is used as an optimizer. The optimizer 'sgd' is preferred due to ability of fast convergence. The values 0.02 and 0.8 are used as learning rate and momentum parameters respectively. The DNN model is learned to optimize the cross entropy function. The training is continued for 100 iterations. The size of training dataset is $70 \%$ and remaining $30 \%$ data samples are used as validation set. After learning, loss and accuracies for training and validation are plotted over the learning steps as shown in Figure 4 and Figure 5. The model shows good fitting since the gap between two plots is low. Table 1 gives the results of this DNN based leaf classification model.

Figure 4. Learning accuracy and validation accuracy plots for experiment with handcraft features and DNN

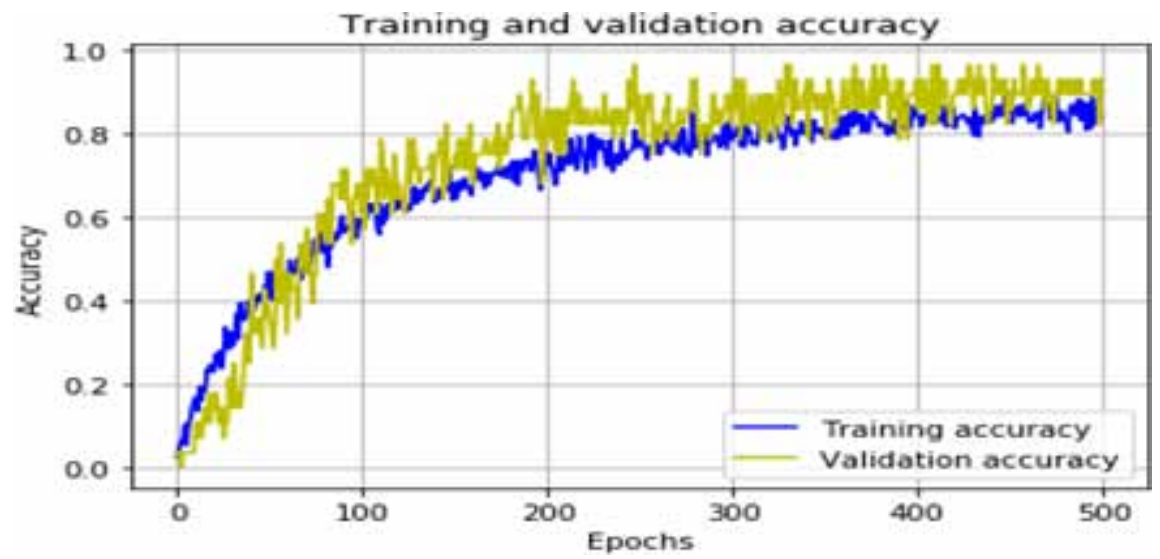

Figure 5. Learning loss and validation loss curves for experiment with handcraft features and DNN

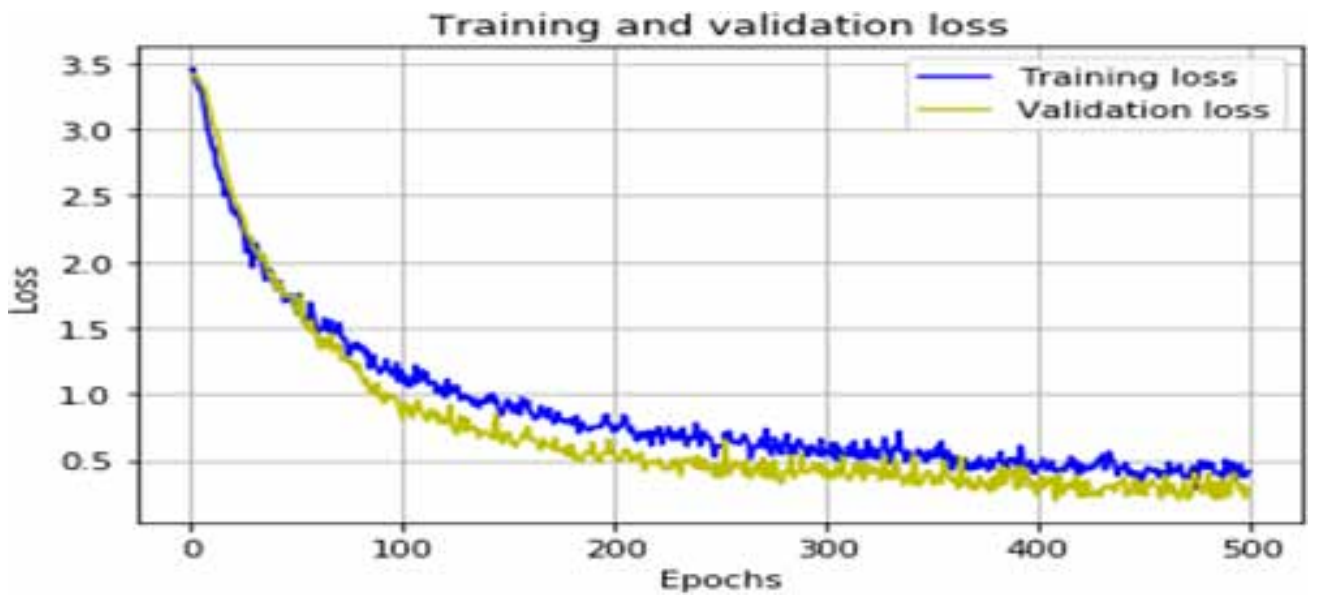


Table 1. Learning parameters

\begin{tabular}{|l|l|}
\hline \multicolumn{1}{|c|}{ Learning Parameter } & \multicolumn{1}{c|}{ Value } \\
\hline Metric & accuracy \\
\hline Loss function & categorical cross entropy \\
\hline Optimizer & adam \\
\hline
\end{tabular}

\subsection{Experiment 2 - Non-Handcraft Features Based Convolution Neural Network Leaf Classification Model}

The deep convolution neural network is designed in this experiment for leaf classification. This CNN model has three convolution layers of size 64, 128 and 128 followed by max-pooling layers of size $2 \times 2$ and two densely connected layers of size 256 . An output layer dense layer of size 30 corresponding to 30 leaf classes is used at last. ReLU as the activation function for hidden layers and sigmoid for the output layer are applied. The standard categorical cross-entropy loss is used to minimize during learning. To avoid overfitting dropout layers are added after each convolution layer. The dropout rate 0.2 gives the best performance. Model is compiled using the Adam optimizer and trained for 100 steps. The model is compiled with the learning parameters as in Table 1. Table 2 provides the learning hyper-parameters used for model. Figure 6 and Figure 7 present the plots for loss and accuracies. The low gap between training and validation curves shows the robustness of the model. The performance measures are given in Table 3.

Table 2. Training hyper-parameters

\begin{tabular}{|l|l|}
\hline \multicolumn{1}{|c|}{ Training Hyperparameter } & \multicolumn{1}{c|}{ Value } \\
\hline Learning rate & 0.001 \\
\hline Epochs & 100 \\
\hline Batch size & 32 \\
\hline Dropout ratio & 0.2 \\
\hline
\end{tabular}

Figure 6. Learning accuracy and validation accuracy plots for experiment with CNN for leaf images

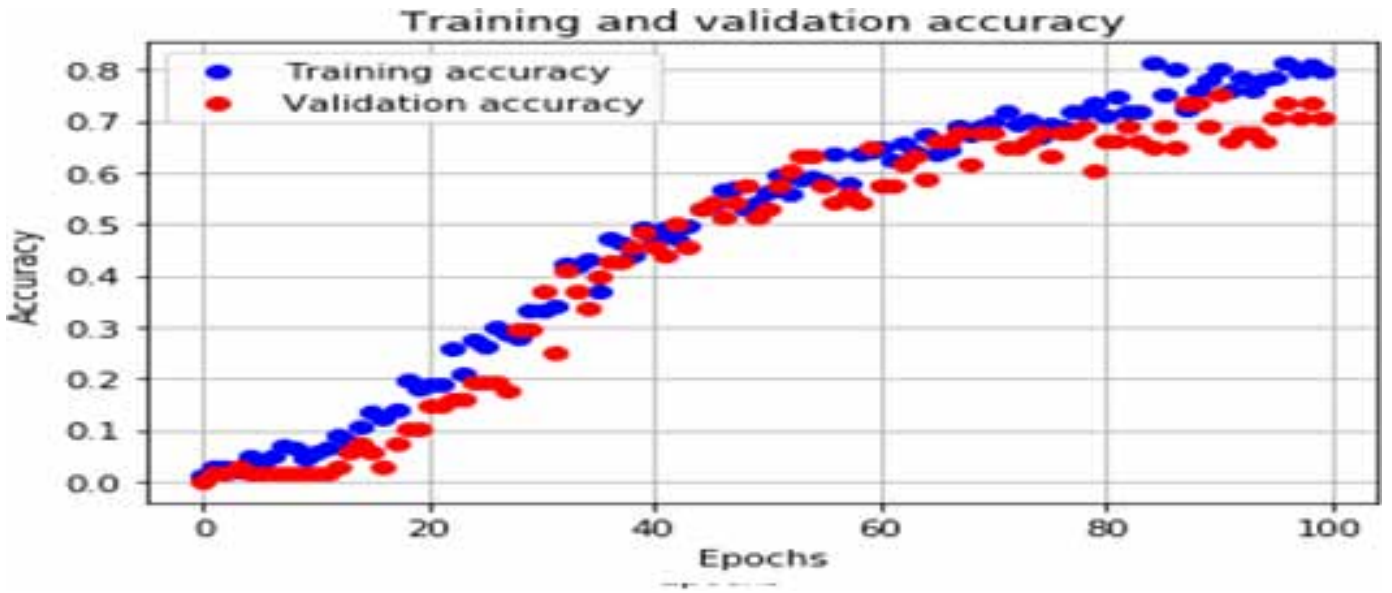


Figure 7. Learning loss and validation loss curves for experiment with CNN for leaf images

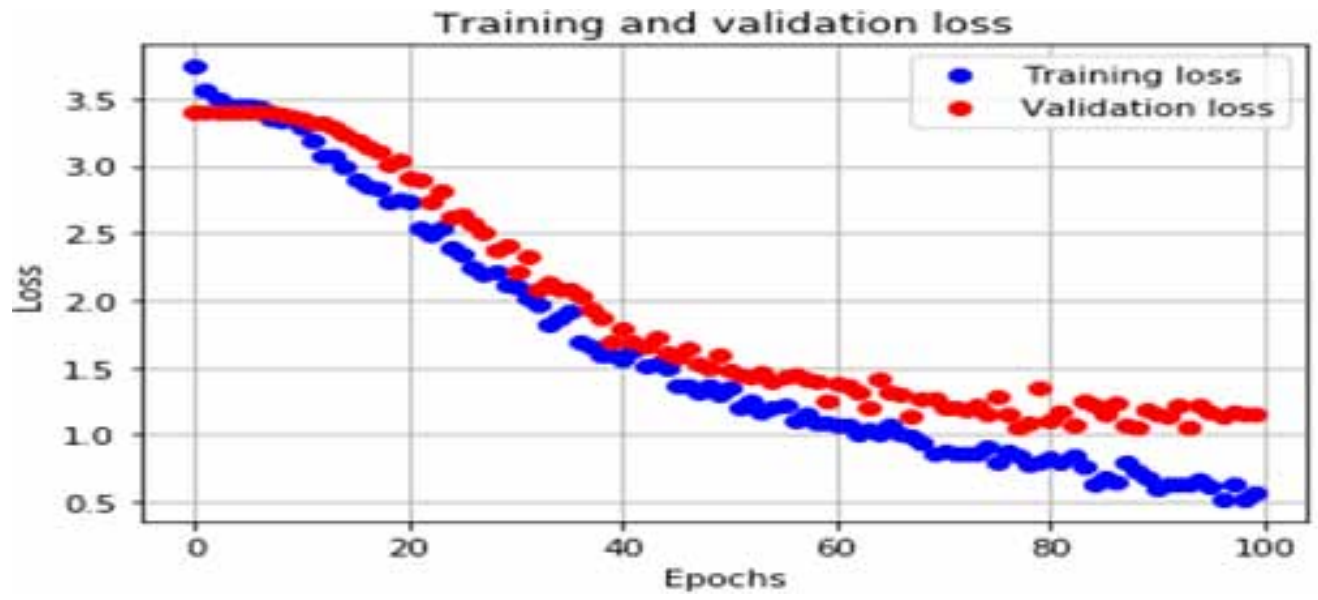

Table 3. Performance of leaf classification models

\begin{tabular}{|c|c|c|c|c|c|c|}
\hline \multirow[t]{2}{*}{ Leaf Class } & \multicolumn{3}{|c|}{$\begin{array}{c}\text { Performance Using Features and DNN } \\
\text { Classification Model }\end{array}$} & \multicolumn{3}{|c|}{$\begin{array}{c}\text { Performance Using CNN Classification } \\
\text { Model }\end{array}$} \\
\hline & Precision & Recall & f1-Score & Precision & Recall & f1-Score \\
\hline Quercus suber & 0.9 & 0.75 & 0.82 & 1 & 1 & 1 \\
\hline Salix atrocinera & 0.88 & 0.7 & 0.78 & 1 & 0.9 & 0.95 \\
\hline Populus nigra & 1 & 0.9 & 0.95 & 1 & 1 & 1 \\
\hline Alnus sp. & 0.8 & 1 & 0.89 & 0.89 & 1 & 0.94 \\
\hline Quercus robur & 1 & 1 & 1 & 0.92 & 1 & 0.96 \\
\hline Crataegus monogyna & 1 & 0.88 & 0.93 & 1 & 1 & 1 \\
\hline Ilex aquifolium & 0.9 & 0.9 & 0.9 & 1 & 0.9 & 0.95 \\
\hline Nerium oleander & 1 & 1 & 1 & 1 & 1 & 1 \\
\hline Betula pubescens & 0.86 & 0.86 & 0.86 & 1 & 0.86 & 0.92 \\
\hline Tilia tomentosa & 1 & 0.85 & 0.92 & 1 & 0.62 & 0.76 \\
\hline Acer palmatum & 1 & 1 & 1 & 1 & 1 & 1 \\
\hline Celtis sp. & 0.92 & 1 & 0.96 & 1 & 1 & 1 \\
\hline Corylus avellana & 0.85 & 0.85 & 0.85 & 0.92 & 0.92 & 0.92 \\
\hline Castanea sativa & 1 & 0.75 & 0.86 & 1 & 0.83 & 0.91 \\
\hline Populus alba & 1 & 1 & 1 & 1 & 1 & 1 \\
\hline Primula vulgaris & 0.92 & 1 & 0.96 & 1 & 1 & 1 \\
\hline Erodium sp. & 0.92 & 1 & 0.96 & 1 & 1 & 1 \\
\hline Bougainvillea sp. & 1 & 0.85 & 0.92 & 0.81 & 1 & 0.9 \\
\hline Arisarum vulgare & 1 & 1 & 1 & 1 & 1 & 1 \\
\hline Euonymus japonicus & 1 & 0.92 & 0.96 & 1 & 0.92 & 0.96 \\
\hline
\end{tabular}


Table 3. Continued

\begin{tabular}{|c|c|c|c|c|c|c|}
\hline \multirow[t]{2}{*}{ Leaf Class } & \multicolumn{3}{|c|}{$\begin{array}{c}\text { Performance Using Features and DNN } \\
\text { Classification Model }\end{array}$} & \multicolumn{3}{|c|}{$\begin{array}{c}\text { Performance Using CNN Classification } \\
\text { Model }\end{array}$} \\
\hline & Precision & Recall & f1-Score & Precision & Recall & f1-Score \\
\hline Ilex perado ssp. azorica & 0.8 & 0.73 & 0.76 & 0.85 & 1 & 0.92 \\
\hline Magnolia soulangeana & 0.73 & 0.67 & 0.7 & 1 & 1 & 1 \\
\hline Buxus sempervirens & 0.86 & 1 & 0.92 & 1 & 1 & 1 \\
\hline Urtica dioica & 1 & 1 & 1 & 0.75 & 1 & 0.86 \\
\hline Podocarpus sp. & 0.92 & 1 & 0.96 & 1 & 1 & 1 \\
\hline Acca sellowiana & 1 & 0.82 & 0.9 & 1 & 1 & 1 \\
\hline Hydrangea sp. & 1 & 1 & 1 & 1 & 0.82 & 0.9 \\
\hline Pseudosasa japonica & 1 & 0.91 & 0.95 & 1 & 1 & 1 \\
\hline Magnolia grandiora & 0.89 & 0.73 & 0.8 & 1 & 0.91 & 0.95 \\
\hline Geranium sp. & 1 & 1 & 1 & 1 & 0.9 & 0.95 \\
\hline Average & 0.94 & 0.90 & 0.92 & 0.97 & 0.95 & 0.96 \\
\hline Average Accuracy & \multicolumn{3}{|l|}{91.17} & \multicolumn{3}{|l|}{95.58} \\
\hline
\end{tabular}

After result analysis, it is evident that CNN leaf classification models perform better than DNN leaf classification model. The average precision, recall, and f1-score metrics are 0.97, 0.95, and 0.96, respectively, for CNN model which are better than DNN model. The average accuracy in case of DNN model is $91.17 \%$ whereas the CNN models gives $95.58 \%$ accuracy.

\section{CONCLUSION}

This work has presented the application of two deep learning classification frameworks for leaf classification. The neural network with more than one hidden layer is referred as deep neural network. The deep neural network model has used shape and texture based handcrafted features for leaf classification. The second classification model is based on convolution neural network. The raw leaf images are passed to CNN for classification. The CNN model has not only shown the better performance in terms of accuracy but also it reduces the overhead of feature extraction. In future, this work can be stretched by considering more leaf classes in the dataset. 


\section{REFERENCES}

Chaki, J., \& Parekh, R. (2011). Plant leaf recognition using shape based features and neural network classifiers. International Journal of Advanced Computer Science and Applications, 2(10). doi:10.14569/IJACSA.2011.021007

Du, J. X., Zhai, C. M., \& Wang, Q. P. (2013). Recognition of plant leaf image based on fractal dimension features. Neurocomputing, 116, 150-156. doi:10.1016/j.neucom.2012.03.028

Ehsanirad, A., \& Kumar, S. (2010). Leaf recognition for plant classification using GLCM and PCA methods. Oriental journal of Computer Science \& technology, 3(1), 31-36.

Elhariri, E., El-Bendary, N., \& Hassanien, A. E. (2014). Plant classification system based on leaf features. In Proceedings of the 2014 9th International Conference on Computer Engineering \& Systems (ICCES) (pp. 271276). IEEE. doi:10.1109/ICCES.2014.7030971

Ferentinos, K. P. (2018). Deep learning models for plant disease detection and diagnosis. Computers and Electronics in Agriculture, 145, 311-318. doi:10.1016/j.compag.2018.01.009

Gaur, P., \& Tiwari, S. (2014). 2D QR barcode recognition using texture features and neural network. International Journal of Research in Advent Technology, 2(5), 433-437.

Haque, F., \& Haque, S. (2018). Plant recognition system using leaf shape features and minimum Euclidean distance. ICTACT Journal on Image \& Video Processing, 9(2).

Kadir, A., Nugroho, L. E., Susanto, A., \& Santosa, P. I. (2013). Neural network application on foliage plant identification.

Le Cun, Y., Bengio, Y., \& Hinton, G. (2015). Deep learning. Nature, 521(7553), 436-444. doi:10.1038/ nature14539 PMID:26017442

Lee, S. H., Chan, C. S., Mayo, S. J., \& Remagnino, P. (2017). How deep learning extracts and learns leaf features for plant classification. Pattern Recognition, 71, 1-13. doi:10.1016/j.patcog.2017.05.015

Liu, S., Chen, W., \& Dong, X. (2018). Automatic Classification of Chinese Herbal Based on Deep Learning Method. In Proceedings of the 2018 14th International Conference on Natural Computation, Fuzzy Systems and Knowledge Discovery (ICNC-FSKD) (pp. 235-238). IEEE. doi:10.1109/FSKD.2018.8687165

Priya, C. A., Balasaravanan, T., \& Thanamani, A. S. (2012). An efficient leaf recognition algorithm for plant classification using support vector machine. In Proceedings of the International conference on pattern recognition, informatics and medical engineering (PRIME-2012) (pp. 428-432). IEEE. doi:10.1109/ICPRIME.2012.6208384

Raal, A., \& Sõukand, R. (2005). Classification of Remedies and Medical Plants of Estonian Ethnopharmacology. Trames Journal of the Humanities and Social Sciences, 9(3).

Riaz, A., Farhan, S., Fahiem, M. A., \& Tauseef, H. (2018). An Ensemble Classifier based Leaf Recognition Approach for Plant Species Classification using Leaf Texture, Morphology and Shape. The Nucleus, 55(1), 1-7. PMID:28934014

Shin, H. C., Roth, H. R., Gao, M., Lu, L., Xu, Z., Nogues, I., \& Summers, R. M. (2016). Deep convolutional neural networks for computer-aided detection: $\mathrm{CNN}$ architectures, dataset characteristics and transfer learning. IEEE Transactions on Medical Imaging, 35(5), 1285-1298. doi:10.1109/TMI.2016.2528162 PMID:26886976

Silva, P. F., Marcal, A. R., \& da Silva, R. M. A. (2013). Evaluation of features for leaf discrimination. In Proceedings of the International Conference Image Analysis and Recognition (pp. 197-204). Springer. doi:10.1007/978-3-642-39094-4_23

Tan, J. W., Chang, S. W., Kareem, S. B. A., Yap, H. J., \& Yong, K. T. (2018). Deep Learning for Plant Species Classification using Leaf Vein Morphometric. IEEE/ACM Transactions on Computational Biology and Bioinformatics. doi:10.1109/TCBB.2018.2848653 PMID:29994129

Tiwari, S. (2017). A Pattern Classification Based approach for Blur Classification. Indonesian Journal of Electrical Engineering and Informatics, 5(2), 162-173.

Tiwari, S. (2018). An Analysis in Tissue Classification for Colorectal Cancer Histology Using Convolution Neural Network and Colour Models. International Journal of Information System Modeling and Design, 9(4), 1-19. doi:10.4018/IJISMD.2018100101 